\title{
Prise en charge des patientes et patients en fin de vie
}

\author{
Directives médico-éthiques de I'ASSM. La version allemande est la version d'origine
}

\section{Préambule}

Souvent, les personnes se trouvant dans la phase ultime de leur vie ont un besoin particulier d'aide et de protection. Elles nous rappellent que toute existence humaine a une fin. Les décisions relatives à la fin de la vie posent de grands défis au patient lui-même, bien sûr, mais aussi à ses proches, aux médecins et à l'équipe responsable de sa prise en charge. Le but de ces directives est de mettre en évidence les tâches, les possibilités et les limites de la prise en charge des patients en fin de vie. L'objectif de base consiste à soulager les souffrances et à offrir au patient la meilleure qualité de vie possible ainsi qu'un soutien à ses proches.

Contrairement à la dernière version des directives de 1995, les dispositions qui suivent portent uniquement sur la situation des patients mourants. Les directives médico-éthiques pour le traitement et la prise en charge des patients souffrant d'atteintes cérébrales extrêmes de longue durée ont fait pour la première fois l'objet de directives distinctes. Cependant, comme il existe toute une série de questions et problèmes communs à ces deux types de situations, il convient de souligner l'importance de ces autres directives pour les questions abordées ci-après. C'est le cas également pour les Directives concernant les problèmes éthiques aux soins intensifs et des nouvelles Directives sur le traitement et la prise en charge des personnes âgées en situation de dépendance.

Concernant la problématique particulière des très grands prématurés, nous renvoyons aux Recommandations de la Société Suisse de Néonatologie concernant la prise en charge des prématurés à la limite de la viabilité.

\section{Directives}

\section{Champ d'application}

Ces directives concernent la prise en charge des patients en fin de vie. Il s'agit de malades pour lesquels le médecin, se fondant sur des signes cliniques, a acquis la conviction que s'est installé un processus dont on sait par expérience qu'il entraîne la mort en l'espace de quelques jours ou de quelques semaines.

Les mêmes critères s'appliquent aux nouveau-nés, aux enfants et aux adolescents en fin de vie; dans la mesure où, pour ces patients pédiatriques, des aspects particuliers sont à prendre en compte, il en est fait état dans les paragraphes correspondants.

\section{Droit à I'autodétermination}

Chaque patient a le droit de disposer librement de sa personne. L'information du patient ou de son représentant sur la situation médicale doit être précoce, complète et compréhensible, afin de permettre un choix éclairé. Cela suppose qu'une communication sensible et ouverte s'installe, et que le médecin soit prêt à informer tant des possibilités et limites de l'action curative que des soins palliatifs.

\subsection{Patients capables de discernement}

Le respect de la volonté des patients capables de discernement est fondamental dans l'action médicale. C'est pourquoi, un traitement médical contre la volonté exprimée du patient capable de discernement est inadmissible. Ceci vaut également lorsque, du point de vue des tiers, cette volonté semble aller à l'encontre des intérêts bien compris du patient. Des mineurs ou des personnes sous tutelle peuvent également être capables de discernement lorsqu'il s'agit de consentir à un traitement.

\subsection{Patients incapables de discernement}

\subsubsection{Recherche de la volonté présumée}

Si le patient n'est plus en état d'exprimer sa volonté, sa volonté présumée doit être recherchée. C'est pourquoi, le médecin ou le personnel soignant doit déterminer si le patient a rédigé des directives anticipées, s'il a désigné par procuration un représentant thérapeutique ou s'il a clairement exprimé sa volonté à ses proches. Il convient en outre de vérifier si un représentant légal a été désigné. Si tel est le cas, il faut rechercher leur consentement. 
Directives anticipées: Tout patient peut prendre à l'avance des dispositions en ce qui concerne le traitement qu'il désire recevoir, cela pour le cas où il ne serait plus capable de discernement (directives anticipées). La volonté du patient doit être respectée, dans la mesure où elle est pertinente pour la situation concrète et pour autant qu'il n'existe pas d'indices permettant de penser qu'elle puisse s'être modifiée entre-temps.

Représentant mandaté pour les affaires médicales: Tout patient peut déterminer à l'avance et par écrit un «représentant mandaté pour les affaires médicales» (appelé ci-après «représentant thérapeutique») qui peut, à sa place, consentir à un traitement, à partir du moment où il deviendrait incapable de discernement. La décision doit être prise en tenant compte d'une éventuelle directive anticipée et en accord avec le représentant thérapeutique désigné.

Autres indices de la volonté présumée: Il n'est pas rare qu'il n'existe ni directives anticipées, ni représentant thérapeutique, et qu'aucun représentant légal n'ait été désigné. Dans ces situations, des informations sur les convictions et comportements antérieurs du patient doivent être recherchées de manière ciblée. A cet égard, une attention particulière doit être portée aux discussions avec les proches et d'éventuelles autres personnes (par ex. médecin de famille).

\subsubsection{Recherche de l'intérêt bien compris du patient}

Toutefois, il est parfois impossible de trouver des indices concernant la volonté présumée, par ex. lorsqu'il n'y a pas de proches ou lorsque l'urgence de la situation ne laisse pas le temps de consulter des tierces personnes. Dans de telles situations, la décision du médecin traitant doit être prise en accord avec les intérêts bien compris du patient.

Pour les enfants et adolescents incapables de discernement, c'est en principe la volonté du représentant légal qui est déterminante; il s'agit généralement des parents. Toutefois, les décisions touchant à la vie et à la mort d'un enfant représentent souvent un fardeau trop lourd pour les parents, qui sont alors incapables de trancher seuls. Ils ne devraient donc être associés au processus décisionnel, que dans la mesure où ils le souhaitent. Les décisions relatives au traitement et à la prise en charge doivent être prises dans l'intérêt bien compris de l'enfant et en accord avec ses parents ou son représentant légal.

\subsubsection{Situations conflictuelles}

Si les représentants (légal ou thérapeutique) rejettent une mesure qui du point de vue médical est indiscutablement dans l'intérêt du patient incapable de discernement, toutes les possibilités d'intervention, comme par ex. l'intervention d'un conseil d'éthique, doivent avoir été épuisées. En l'absence d'accord, il sera fait appel à l'autorité tutélaire. En situation d'urgence, lorsque le temps pour ces étapes fait défaut, on peut introduire une mesure même contre la volonté du représentant.

\section{Traitement et prise en charge}

\subsection{Soins palliatifs}

Les patients dans la dernière phase de leur vie ont droit à une prise en charge palliative. Celle-ci comprend l'ensemble des traitements médicaux et des soins, ainsi qu'un appui psychique, social et spirituel aux patients et à leurs proches, le but étant de soulager les souffrances et d'assurer la meilleure qualité de vie possible du patient.

La tâche principale de l'équipe responsable de la prise en charge du patient est le traitement efficace des symptômes, y compris la gestion de la détresse ainsi que la disponibilité et l'accompagnement du patient et de ses proches. Toutes les ressources techniques et humaines potentiellement utiles (par ex. professionnels de l'accompagnement psychologique, social et spirituel) doivent être sollicitées en cas de besoin. La prise en charge palliative doit être proposée suffisamment tôt et là où se trouve le patient (à l'hôpital, dans une institution ou à domicile).

Le médecin doit soulager les douleurs et souffrances, même si, dans certains cas, cela peut avoir une influence sur la durée de la vie (raccourcissement ou prolongation). Face à des symptômes résistant aux traitements, une sédation palliative peut parfois se révéler nécessaire. A cet égard, il s'agit de veiller à ce que la sédation ne dépasse pas le niveau nécessaire au soulagement des symptômes.

Les décisions concernant le traitement et la prise en charge doivent, dans toute la mesure du possible, être soutenues par l'équipe responsable de la prise en charge du patient et de ses proches.

Il est important d'associer les proches, en reconnaissant leur double rôle de personnes accompagnantes et accompagnées. Les souhaits personnels concernant le déroulement de la dernière phase de la vie doivent être respectés. La prise en charge doit aussi inclure un accompa- 
gnement des proches, qui, dans certains cas, peut se prolonger au-delà de la mort du patient. Il faut veiller à traiter un défunt avec respect et accorder, dans la mesure du possible, l'espace nécessaire aux rituels culturels et religieux de la famille.

\subsection{Abstention ou retrait thérapeutique}

Une fois que le processus de la mort est engagé, le renoncement à des mesures destinées à préserver la vie ou la suspension de telles mesures peut être justifié, voire même indiqué. Dans la prise de décision, des critères comme le pronostic, les bénéfices thérapeutiques prévisibles en termes de qualité de vie, ainsi que les contraintes liées à la thérapie proposée, jouent un rôle.

Les mêmes considérations s'appliquent en principe aux nouveau-nés, aux nourrissons et aux enfants en bas âge. Le fait qu'avec ces catégories de patients pédiatriques, on ne puisse pas se fonder sur une volonté présumée ou sur la personnalité, complique toutefois la prise de décision. Le recours à des mesures lourdes visant à maintenir les fonctions vitales doit donc dépendre principalement du pronostic. Pour ce faire, il importe de mettre en balance, d'une part, les contraintes liées à la thérapie en termes de douleurs, de malaises et de limitation, et, d'autre part, le bénéfice que l'on peut en attendre en termes de bien-être, de possibilités relationnelles et de conscience du vécu.

\section{Limites de I'activité médicale}

Le respect de la volonté du patient atteint ses limites quand un patient réclame des mesures qui sont inefficaces ou inappropriées ou qui sont incompatibles avec les valeurs morales personnelles du médecin, la déontologie ou le droit en vigueur.

\subsection{Assistance au suicide}

Aux termes de l'article 115 du code pénal, l'assistance au suicide n'est pas punissable lorsqu'elle intervient sans mobile égoïste. Ce principe s'applique à tout individu.

La mission des médecins prenant en charge des patients en fin de vie consiste à soulager et accompagner le patient. Il n'est pas de leur devoir de proposer une assistance au suicide, au contraire, ils ont le devoir de soulager les souffrances qui pourraient être à l'origine d'un désir de suicide. Toutefois, un patient en fin de vie ne supportant plus sa situation peut exprimer son désir de mourir et persister dans ce désir.
Dans ce genre de situation aux confins de la vie et de la mort, le médecin peut se retrouver face à un conflit difficile à gérer. D'une part, l'assistance au suicide ne fait pas partie de l'activité médicale, car elle est contraire aux buts de la médecine. D'autre part, le respect de la volonté du patient est fondamental dans la relation médecin-patient. Un tel dilemme exige une décision morale personnelle du médecin qui doit être respectée en tant que telle. Le médecin a, dans tous les cas, le droit de refuser d'apporter une aide au suicide. Si toutefois, dans des situations exceptionnelles, il accepte d'apporter une aide au suicide à un patient, il lui incombe la responsabilité de vérifier si les exigences minimales suivantes sont réunies:

- La maladie dont souffre le patient permet de considérer que la fin de la vie est proche.

- Des alternatives de traitements ont été proposées et, si souhaitées par le patient, mises en œuvre.

- Le patient est capable de discernement. Son désir de mourir est mûrement réfléchi, il ne résulte pas d'une pression extérieure et il est persistant. Cela doit avoir été vérifié par une tierce personne, qui ne doit pas nécessairement être médecin.

Le dernier geste du processus conduisant à la mort doit dans tous les cas être accompli par le patient lui-même.

\subsection{Meurtre à la demande de la victime}

Le médecin doit refuser de mettre fin à la vie d'un patient, même sur demande sérieuse et instante. Le meurtre à la demande de la victime est punissable selon l'article 114 du code pénal.

\section{Commentaires}

\section{ad 1. (Champ d'application)}

D'après cette définition, les patients en fin de vie doivent être distingués des patients souffrant de maladies incurables et évolutives dans la mesure où cette évolution peut s'étendre sur des mois voire des années. Par «signes cliniques» on entend l'ensemble des observations, comme par exemple la dégradation des fonctions vitales, des résultats objectivement défavorables et l'évaluation de l'état général, qui caractérisent le début du processus de la mort. Il convient toutefois de souligner qu'il n'est pas rare que les décisions médicales d'interruption ou de renoncement à un traitement coïncident avec le début du processus de la mort, de telle façon qu'il existe toujours une certaine zone d'ombre. 


\section{ad 2.1 (Patients capables de discernement)}

Les critères suivants aident à déterminer la capacité de discernement selon l'art. 16 du Code Civil:

- la capacité de comprendre les informations relatives aux décisions à prendre;

- la capacité d'évaluer correctement une situation et les conséquences découlant des différentes options envisageables;

- la capacité d'évaluer rationnellement une information dans le contexte d'un système de valeur cohérent;

- la capacité de faire librement ses propres choix.

Le discernement s'apprécie par rapport à un acte déterminé (en fonction de la nature et de la complexité de cet acte). Il doit exister au moment où la décision est prise.

\section{ad 2.2 (Patients incapables de discernement)}

La question du consentement $d u$ patient incapable de discernement qui n'a pas de représentant légal et qui n'a pas désigné de représentant thérapeutique n'est pas expressément réglée dans la législation fédérale. On trouve toutefois des dispositions dans les législations sanitaires cantonales. Mais la question n'est pas réglée de manière uniforme. Cf. à ce sujet: «Traitement et prise en charge des personnes âgées en situation de dépendance, directives médico-éthiques et recommandations», note 6 en bas de page).

\section{ad 2.2.1 (Recherche de la volonté présumée)}

La volonté présumée correspond à la volonté que le patient exprimerait vraisemblablement s'il était encore capable de discernement. Elle résulte de l'évaluation de toutes les informations à disposition, telles que directives anticipées, désignation d'un représentant thérapeutique, déclarations faites antérieurement et autres indices biographiques.

Les proches au sens des présentes directives sont les personnes proches du patient, en particulier le conjoint ou le ou la partenaire, les enfants ou les parents et frères et sœurs.

\section{ad 2.2.2 (Recherche de l'intérêt bien compris du patient)}

Par «Recherche de l'intérêt bien compris du patient», on entend l'introduction de mesures médicales ou de soins qui semblent indiquées, et auxquelles un patient présumé raisonnable consentirait vraisemblablement, dans une telle situation.

\section{ad 2.2.3 (Situations conflictuelles)}

Bien que les proches n'aient aucun droit de décision, on essaiera de trouver un consensus en cas de conflit.

\section{ad 3.1 (Soins palliatifs)}

\section{Limites de la médecine palliative}

Toute souffrance liée à l'agonie et à la mort n'est pas forcément évitable. Identifier certaines limites et y faire face fait partie intégrante de la prise en charge du patient et de ses proches. Si une situation particulièrement difficile pèse trop lourd sur l'équipe soignante, on peut faire appel à un soutien professionnel externe.

\section{Influence sur la durée de vie}

L' «effet accélérateur» de substances actives sur la fin de la vie a été pendant longtemps surestimé. En général, les analgésiques et les sédatifs, quand ils sont employés correctement et dans le but exclusif de contrôler les symptômes dans la dernière semaine de vie, n'entraînent pas un raccourcissement du temps de survie.

Les analgésiques et les sédatifs peuvent également être employés de manière abusive, pour provoquer le décès. Mais, dans la majorité des cas, on peut déjà, selon le dosage ou l'augmentation de la dose de médicaments, faire une différence entre l'atténuation de la douleur et des symptômes à des fins palliatives, et l'abrégement intentionnel de la vie.

\section{Formation postgraduée et formation continue}

La prise en charge de patients en fin de vie présuppose des connaissances et compétences dans le domaine de la médecine, des soins et de l'accompagnement palliatifs. 


\section{ad 3.2 (Abstention ou arrêt thérapeutique)}

Les mesures destinées à préserver la vie comportent en particulier la réhydratation et l'alimentation artificielle, la respiration assistée et la réanimation cardiopulmonaire. En fonction de la situation, l'administration d'oxygène, une médication, une transfusion sanguine, une dialyse ou une intervention chirurgicale doivent également être discutées.

\section{ad 4.1 (Assistance au suicide)}

En ce qui concerne l'attitude face à une demande de suicide dans des institutions de soins, il s'agit d'observer les directives et recommandations pour le traitement et la prise en charge des personnes âgées en situation de dépendance.

Les dirigeants peuvent interdire l'assistance au suicide à leurs collaborateurs; par contre, ils ne peuvent pas les y contraindre.

Le processus décisionnel conduisant à l'assistance au suicide ou à son refus doit être documenté.

Un décès survenant suite à une aide au suicide doit être déclaré aux autorités comme décès pour cause non naturelle. Le médecin ayant prêté son assistance à un suicide ne doit pas remplir lui-même le certificat de décès.

\section{Recommandations à I'intention des autorités sanitaires compétentes}

\section{Ressources}

Malgré les ressources limitées à disposition, les responsables du système de santé devraient avoir une politique garantissant que tous ces patients en fin de vie puissent bénéficier d'une prise en charge palliative au sens des présentes directives.

Les institutions devraient être chargées et avoir la possibilité de mettre à disposition toutes les conditions nécessaires comme les locaux, les ressources personnelles, l'accompagnement de l'équipe de soins dans les situations difficiles, etc.

\section{Formation prégraduée et postgraduée}

Les contenus de la médecine palliative doivent être intégrés à la formation prégraduée, postgraduée et continue de toutes les catégories professionnelles participant à la prise en charge des patients.

\section{Membres de la sous-commission responsable de l'élaboration de ces directives}

Dr théol. Markus Zimmermann-Acklin, Lucerne, Président; PD Dr phil. Jürg Bernhard, Berne; Dr méd. Georg Bosshard, Zurich; Ulrike Büchs, Pasteur, Winterthur; Christine Champion, infirmière, Moudon; Dr méd. Daniel Grob, Zurich; Prof. Dr méd. Christian Kind, St-Gall; Dr méd. Hans Neuenschwander, Lugano; Prof. Dr méd. Rudolf Ritz, Bâle; lic. iur. Michelle Salathé, Bâle (ex officio); Elisabeth Spichiger, infirmière, Berne; Dr méd. Philipp Weiss, Bâle; Prof. Dr méd. Michel Vallotton, Genève, Président CCE (ex officio).

\section{La sous-commmission remercie les experts} suivants pour leurs conseils judicieux Dr méd. Klaus Bally, Bâle; Prof. Dr méd. Verena Briner, Lucerne; Prof. Dr théol. Johannes Fischer, Zurich; Hanspeter Kuhn, avocat, Berne; lic. theol. Settimio Monteverde, Bâle; Catherine Panchaud, M. Sc, Puidoux; PD Dr phil. Klaus Peter Rippe, Zurich; Prof. Dr iur. et Dr h.c. Kurt Seelmann, Bâle; Prof. Dr méd. Frédéric Stiefel, Lausanne; Prof. Dr méd. Andreas Stuck, Berne.

\section{Approbation}

Approuvées par le Sénat de l'ASSM, le 25 novembre 2004 .

Prof. Michel Vallotton, Genève, Président de la Commission centrale d'éthique de l'ASSM

\section{Directives citées}

- Traitement et prise en charge des patients souffrant d'atteintes cérébrales extrêmes de longue durée. Directives médico-éthiques (2004).

- Traitement et prise en charge des personnes âgées en situation de dépendance. Directives et recommandations médico-éthiques (2004).

- Problèmes éthiques aux soins intensifs. Directives médico-éthiques (1999).

- Société Suisse de Néonatologie. Recommandations pour la prise en charge des prématurés à la limite de la viabilité (22-26 semaines de gestation). Bull Méd Suisses 2002;83 (29/30):1586-602. Paediatrica 2002;13(2): 34-41. 